\title{
Estimates of five restricted partition functions that are quasi polynomials
}

\author{
A. David Christopher · M. Davamani Christober
}

Received: 12 October 2013 / Revised: 16 June 2014 / Accepted: 17 June 2014 /

Published online: 28 June 2014

(C) The Author(s) 2014. This article is published with open access at SpringerLink.com

Abstract A function $f$ defined on $\mathbb{N}$ is said to be a quasi polynomial if, $f(\alpha n+r)$
is a polynomial in $n$ for each $r=0,1, \ldots, \alpha-1$, where $\alpha$ is a positive inte-
ger. In this article, we show that the below given restricted partition functions are
quasi polynomials: (i) $a(n, k)$-number of partitions of $n$ with exactly $k$ parts and
least part being less than $k$, (ii) $a q(n, k)$-number of distinct partitions (partitions
with distinct parts) of $n$ with exactly $k$ parts and least part being less than $k$, (iii)
$L e(n, k, m)$-number of partitions of $n$ with exactly $k$ parts and $m$ least parts, (iv)
$L a(n, k, 1)$-number of partitions of $n$ with exactly $k$ parts and one largest part and (v)
$d(n, k)$-number of partitions of $n$ with exactly $k$ parts and difference between least
part and largest part exceeds $k-2$. Consequently, following estimates were derived: (i)

$$
a(n, k) \sim \frac{n^{k-2}}{(k-2) !^{2}}
$$

(ii)

$$
a q(n, k) \sim \frac{n^{k-2}}{(k-2) !^{2}}
$$

Communicated by Ari Laptev.

A. D. Christopher $(\varangle) \cdot$ M. D. Christober

Department of Mathematics, The American College, Madurai 625002, Tamil Nadu, India

e-mail: davchrame@yahoo.co.in

M. D. Christober

e-mail: jothichristopher@yahoo.com 
(iii)

$$
\operatorname{Le}(n, k, m) \sim \frac{(k-1) !}{(k-m) !(k-m-2) !} n^{k-m-1}
$$

(iv)

$$
L a(n, k, 1) \sim \frac{n^{k-1}}{k !(k-1) !}
$$

(v)

$$
d(n, k) \sim \frac{n^{k-1}}{k !(k-1) !}
$$

Keywords Restricted partitions · Quasi polynomials · Estimates

Mathematics Subject Classification (2010) Primary 05A17; Secondary 11P82

\section{Introduction}

A function $f$ defined on $\mathbb{N}$ is said to be a quasi polynomial if, $f(\alpha n+r)$ is a polynomial in $n$ for each $r=0,1, \ldots, \alpha-1$, where $\alpha$ is a positive integer. In such case, $\alpha$ is called quasi period of $f$ and we term $f(\alpha n+r)$ as constituent polynomial of $f$.

The notion of quasi polynomial seems to be subsist from the time of Bell [1], who proved that the partition function, $p_{A}(n)$-number of partitions of $n$ with parts from a finite set of positive integers $A$, is a quasi polynomial with each constituent polynomial being of degree at most $|A|-1$ and quasi period being a positive common multiple of elements of A. This fact was also proved recently by Rødseth and Sellers [5].

In this article, we consider partition functions mentioned in the following definition and we show that they are quasi polynomials. This characteristic found in defined functions is impetus in deriving its estimates. The method followed in this article for deriving estimates can be adopted for the other functions which are not considered in this article provided they meet the requisite for derivation.

Definition 1.1 We recall that, a partition of a positive integer $n$ is a non increasing sequence of positive integers say $\pi=\left(a_{1}, a_{2}, \ldots, a_{k}\right)$ such that $\sum_{i=1}^{k} a_{i}=n$. Each $a_{i}$ is called a part of the partition $\pi$. If $a_{i} \neq a_{j} \forall i \neq j$, then $\pi$ is said to be a distinct partition. The partition function, $p(n)$, counts the number of partitions of $n$. The enumerative function which counts a class of partitions that have a fixed number of parts is usually called a restricted partition function.

(i) The function $a(n, k)$ is defined to be the number of partitions of $n$ with exactly $k$ parts and least part being less than $k$, when $n \geq k$. We define $a(n, k)=0$, when $n<k$.

(ii) The function $a q(n, k)$ is defined to be the number of distinct partitions of $n$ with exactly $k$ parts and least part being less than $k$, when $n \geq \frac{k(k+1)}{2}$. We define $a q(n, k)=0$, when $n<\frac{k(k+1)}{2}$. 
(iii) The function $L e(n, k, m)$ is defined to be the number of partitions of $n$ with exactly $k$ parts and $m$ least parts, when $n \geq 2 k-m$. And $L e(n, k, m)=0$ otherwise.

(iv) The function $L a(n, k, M)$ is defined to be the number of partitions of $n$ with exacly $k$ parts and $M$ largest parts, when $n \geq M+k$. And $L a(n, k, M)=0$ otherwise.

(v) The function $d(n, k)$ is defined to be the number of partitions of $n$ with exactly $k$ parts and difference between least part and largest part exceeds $k-2$.

\section{Estimates}

In this section, we derive estimates for the aforementioned functions.

\subsection{Pattern of derivation}

First, we paraphrase the current method of calculating estimate. If $f$ is a quasi polynomial with quasi period $\alpha$ and each constituent polynomial say $f(\alpha l+r)$ is a polynomial in $l$ of degree $k$ having identical leading coefficient say $c(k)$, then

$$
\lim _{l \rightarrow \infty} \frac{f(\alpha l+r)}{(\alpha l+r)^{k}}=\frac{c(k)}{\alpha^{k}} \forall r=0,1, \ldots, \alpha-1 .
$$

Since the limit is valid for each $r=0,1, \ldots, \alpha-1$, we have

$$
\lim _{n \rightarrow \infty} \frac{f(n)}{n^{k}}=\frac{c(k)}{\alpha^{k}}
$$

Equivalently,

$$
f(n) \sim \frac{n^{k} c(k)}{\alpha^{k}}
$$

In order to make use of the above limit process, we need to show that the leading coefficients of all constituent polynomials of the functions that we have considered are identical.

\subsection{Main results}

Theorem 2.1 We have

(i)

$$
a q(n, k) \sim \frac{n^{k-2}}{(k-2) !^{2}}
$$

(ii)

$$
a(n, k) \sim \frac{n^{k-2}}{(k-2) !^{2}} .
$$


Proof As first step of this proof, we establish the following relations:

(i)

$$
q(n, k)=q(n-k, k)+q(n-k, k-1) \quad \text { when } n \geq \frac{k(k+1)}{2},
$$

where $q(n, k)$ is defined to be the number of distinct partitions of $n$ with exactly $k$ parts.

(ii)

$$
a q(n, k)=\sum_{r=1}^{k-1} q(n-r k, k-1) \quad \text { when } n \geq \frac{3 k(k-1)}{2} .
$$

(iii)

$$
a(n, k)=a q\left(n+\frac{k(k-1)}{2}, k\right) \quad \text { when } n \geq k .
$$

Let $q_{n, k, 1}$ and $q_{n, k, \geq 2}$, respectively, be the number of distinct partitions of $n$ with exactly $k$ parts and least part being one, and the number of distinct partitions of $n$ with exactly $k$ parts and least part being greater than one. We notice that, the mapping

$$
\left(a_{1}, a_{2}, \ldots, a_{k}\right) \rightarrow\left(a_{1}-1, a_{2}-1, \ldots, a_{k}-1\right)
$$

establishes one to one correspondence between the following two sets:

- The set of all distinct partitions of $n$ with exactly $k$ parts and least part being greater than one.

- The set of all distinct partitions of $n-k$ with exactly $k$ parts.

Thus, we have $q_{n, k, \geq 2}=q(n-k, k)$. Further, we notice that, the mapping

$$
\left(a_{1}, a_{2}, \ldots, a_{k-1}\right) \rightarrow\left(a_{1}+1, a_{2}+1, \ldots, a_{k-1}+1,1\right)
$$

establishes one to one correspondence between the following two sets:

- The set of all distinct partitions of $n-k$ with exactly $k-1$ parts.

- The set of all distinct partitions of $n$ with exactly $k$ parts and least part being one.

Thus, we have $q_{n, k, 1}=q(n-k, k-1)$. Since $q(n, k)=q_{n, k, 1}+q_{n, k, \geq 2}$; we get the relation (2.3).

We notice that, the mapping

$$
\left(a_{1}, a_{2}, \ldots, a_{k-1}, a_{k}\right) \rightarrow\left(a_{1}+k-1, a_{2}+k-2, \ldots, a_{k-1}+1, a_{k}\right)
$$

establishes one to one correspondence between the following two sets:

- The set of all partitions of $n$ with exactly $k$ parts and least part being less than $k$.

- The set of all distinct partitions of $n+\frac{(k-1) k}{2}$ with exactly $k$ parts and least part being less than $k$.

Thus the relation (2.5) follows. 
Let $r$ be a positive integer such that $r \leq k-1$ and let $q_{r}(n, k)$ be the number of distinct partitions of $n$ with exactly $k$ parts and least part being $r$. We notice that, the mapping

$$
\left(a_{1}, a_{2}, \ldots, a_{k-1}, r\right) \rightarrow\left(a_{1}-r, a_{2}-r, \ldots, a_{k-1}-r\right)
$$

establishes one to one correspondence between the following sets:

- The set of all distinct partitions of $n$ with exactly $k$ parts and least part being $r$.

- The set of all distinct partitions of $n-r k$ with exactly $k-1$ parts.

provided $n \geq \frac{(k-1) k}{2}+(k-1) k$. Accordingly, we have the relation $q_{r}(n, k)=q(n-$ $r k, k-1)$. Since $a q(n, k)=\sum_{r=1}^{k-1} q_{r}(n, k)$; the relation (2.4) follows.

Since $q(n, 1)=1 \forall n \geq 1$, from the relation (2.3) it follows inductively that: $q(k ! l+$ $r, k)$ is a polynomial of degree $k-1$ for every $r=0,1, \ldots, k !-1$. Consequently, from the relations (2.4) and (2.5) it follows that $a q(k ! l+r, k)$ and $a(k ! l+r, k)$ are polynomials, each of which is of degree $k-2$ for every $r=0,1,2, \ldots, k !-1$. Now, we show that the leading coefficient of $q(k ! l+r, k)$ is $\frac{k !^{k-2}}{(k-1) !}$. We adopt proof by induction on $k$. Since the leading coefficient of the polynomial $q(1 ! l+0,1)$ and $q(1 ! l+1,1)$ is 1 , the aforesaid assertion is true for $k=1$.

Assume that, the assertion is true up to some $k-1 \geq 1$. Let $a_{k-1}$ be the leading coefficient of $q(k ! l+r, k)$ for every $0 \leq r \leq k !-1$. We notice that, the leading coefficient of $q(k ! l+r, k)-q(k !(l-1)+r, k)$ is $(k-1) a_{k-1}$. By the relation (2.3), we have

$$
q(k ! l+r, k)-q(k !(l-1)+r, k)=\sum_{i=1}^{(k-1) !} q\left((k-1) !\left(k l+q_{i}\right)+r_{i}, k-1\right) .
$$

Here uniqueness of $\left(r_{i}, q_{i}\right)$ and the bound $0 \leq r_{i} \leq(k-1) !-1$ follows from the relation $r-i k=(k-1) ! q_{i}+r_{i}$ as consequence of Division algorithm. By the induction assumption, we have that: the leading coefficient of each of the polynomial in the right side of the above equality is $\frac{(k-1) !^{k-3} k^{k-2}}{(k-2) !}$. Thus, the leading coefficient of $q(k ! l+r, k)-q(k !(l-1)+r, k)$ is $\frac{k !^{k-2}}{(k-2) !}$. This gives $a_{k-1}=\frac{k !^{k-2}}{(k-1) !}$ as desired.

From the relation (2.4) it follows that

$$
a q(k ! l+r, k)=\sum_{i=1}^{k-1} q\left((k-1) !\left(k l+q_{i}\right)+r_{i}, k-1\right)
$$

Here too uniqueness of $\left(r_{i}, q_{i}\right)$ and the bound $0 \leq r_{i} \leq(k-1) !-1$ follows from the relation $r-i k=(k-1) ! q_{i}+r_{i}$ as consequence of Division algorithm. Thus, the leading coefficient of $a q(k ! l+r, k)$ is $\frac{(k-1) k^{k-2}(k-1) !^{k-3}}{(k-2) !}=(k-1)^{2} k^{k-2}(k-1) !^{k-4}$. Consequently, from the relation (2.5) it follows that the leading coefficient of $a(k ! l+$ $r, k)$ is $(k-1)^{2} k^{k-2}(k-1) !^{k-4}$.

Accordingly, the estimate of both $a(n, k)$ and $a q(n, k)$ is $\frac{n^{k-2}(k-1)^{2} k^{k-2}(k-1) !^{k-4}}{k !^{k-2}}=$ $\frac{n^{k-2}}{(k-2) !^{2}}$, as desired. 
Remark 2.2 (i) In the course of proof it is shown that, the leading coefficient of the constituent polynomial $q(k ! l+r, k)$ is $\frac{k !^{k-2}}{(k-1) !}$ for every $r=0,1, \ldots, k !-1$. Since each constituent polynomial is of degree $k-1$, one can get the following estimate for $q(n, k)$ :

$$
q(n, k) \sim \frac{n^{k-1}}{k !(k-1) !} .
$$

(ii) Using the following easily verifiable identity:

$$
p(n, k)=q\left(n+\frac{k(k-1)}{2}, k\right),
$$

one can see that, the leading coefficient of the constituent polynomial $p(k ! l+r, k)$ is $\frac{k !^{k-2}}{(k-1) !}$ for every $r=0,1, \ldots, k !-1$. Consequently, we have

$$
p(n, k) \sim \frac{n^{k-1}}{k !(k-1) !} .
$$

This estimate is a well established one and number of proofs have been obtained for this. From the generating functions for $p_{\{1,2, \ldots, k\}}(n)$ and $p(n, k)$, one can see that:

$$
p_{\{1,2, \ldots, k\}}(n)=p(n-k, k) .
$$

It is well documented that

$$
p_{A}(n) \sim \frac{n^{|A|-1}}{(|A|-1) ! \prod_{a \in A} a}
$$

when $A$ is a finite set of positive integers with $\operatorname{gcd}(A)=1$. Number of proofs have been obtained for the latter estimate (see [3,4,6-8]). From the relation (2.9), we see that the estimate (2.8) is a particular case of the estimate (2.10).

(iii) If we define $\bar{a}(n, k)$ to be the number of partitions of $n$ with exactly $k$ parts and least part greater than or equal to $k$, then we have:

$$
p(n, k)=a(n, k)+\bar{a}(n, k) .
$$

Since $p(k ! l+r, k)$ is a polynomial of degree $k-1$ and $a(k ! l+r, k)$ is a polynomial of degree $k-2$ for every $r=0,1, \ldots, k !-1$, by just mentioned relation it follows that $\bar{a}(k ! l+r, k)$ is a polynomial of degree $k-1$ with leading coefficient $\frac{k !^{k-2}}{(k-1) !}$ for every $r=0,1, \ldots, k !-1$. Consequently,

$$
\bar{a}(n, k) \sim \frac{n^{k-1}}{k !(k-1) !} .
$$

In similar fashion, if one defines $\overline{a q}(n, k)$ to be the number of distinct partitions of $n$ with exactly $k$ parts and least part being greater than or equal to $k$, then it follows that: 


$$
\overline{a q}(n, k) \sim \frac{n^{k-1}}{k !(k-1) !} .
$$

(iv) In [2] the partition function, $u(n, k)$, is defined to be the number of uniform partitions of $n$ with exactly $k$ parts, and it is shown that:

$$
u(n, k)=\sum_{d|n, d| k} q\left(\frac{n}{d}, \frac{k}{d}\right) .
$$

From this, we have

$$
q(n, k) \leq u(n, k) \leq p(n, k) .
$$

As we have $q(n, k) \sim \frac{n^{k-1}}{k !(k-1) !}$ and $p(n, k) \sim \frac{n^{k-1}}{k !(k-1) !}$, it follows that:

$$
u(n, k) \sim \frac{n^{k-1}}{k !(k-1) !} .
$$

Theorem 2.3 We have

$$
L e(n, k, m) \sim \frac{(k-1) !}{(k-m) !(k-m-2) !} n^{k-m-1}
$$

Proof First, we show that

$$
L e(n, k, m)=L e(n-k, k, m)+p(n-k, k-m) .
$$

We denote a partition say $\pi$ of $n$ by $\pi=\left(a_{1}^{\alpha_{1}} \cdots a_{r}^{\alpha_{r}}\right)$ when there is $\alpha_{i}$ parts of size $a_{i}$; $(i=1,2, \ldots, r)$. Let $\pi=\left(a_{1}^{\alpha_{1}} a_{2}^{\alpha_{2}} \cdots a_{r}^{\alpha_{r}}\right)$ be a partition of $n$ with $\alpha_{1}+\cdots+\alpha_{r}=k$ and $\alpha_{r}=m$, that is, $\pi$ be a partition of $n$ with exactly $k$ parts and $m$ least parts. Now, we enumerate such partitions by considering two cases.

Case (i) Assume $a_{r}>1$. We notice that, the mapping:

$$
\left(a_{1}^{\alpha_{1}} \cdots a_{r}^{\alpha_{r}}\right) \rightarrow\left(\left(a_{1}-1\right)^{\alpha_{1}} \cdots\left(a_{r}-1\right)^{\alpha_{r}}\right)
$$

is a bijection between the following sets:

- The set of partitions of $n$ with exactly $k$ parts, $m$ least parts and least part being greater than 1 .

- The set of partitions of $n-k$ with exactly $k$ parts and $m$ least parts.

We see that, the cardinality of the latter set is $L e(n-k, k, m)$.

Case (ii) Assume $a_{r}=1$. We notice that, the mapping:

$$
\left(a_{1}^{\alpha_{1}} \cdots a_{r}^{\alpha_{r}}\right) \rightarrow\left(\left(a_{1}-1\right)^{\alpha_{1}} \cdots\left(a_{r-1}-1\right)^{\alpha_{r-1}}\right)
$$

is a bijection between the following sets: 
- The set of partitions of $n$ with exactly $k$ parts, $m$ least parts and least part being equal to 1 .

- The set of partitions of $n-k$ with exactly $k-m$ parts.

We see that, the cardinality of the latter set is $p(n-k, k-m)$. Thus the relation (2.14) follows.

From the relation (2.14), one can get inductively that: $L e(k ! l+r, k, m)$ is a polynomial of degree $k-m-1$ for every $r=0,1, \ldots, k !-1$. Now, we prove that, the leading coefficient of $L e(k ! l+r, k, m)$ is $\frac{(k-1) ! k !^{k-m-1}}{(k-m) !(k-m-2) !}$ for every $r=0,1, \ldots, k !-1$. From the relation (2.14) it follows that:

$$
\begin{aligned}
& \operatorname{Le}(k ! l+r, k, m)-\operatorname{Le}(k !(l-1)+r, k, m)=\sum_{i=1}^{(k-1) !} p(k ! l+r-i k, k-m) \\
& =\sum_{i=1}^{(k-1) !} p\left((k-m) !\left(\frac{k !}{(k-m) !} l+q_{i}\right)+r_{i}, k-m\right),
\end{aligned}
$$

where $r_{i}$ and $q_{i}$ were determined uniquely by the relation: $r-i k=(k-m) ! q_{i}+$ $r_{i}$; uniqueness of $\left(r_{i}, q_{i}\right)$ and boundedness of $r_{i}: 0 \leq r_{i} \leq(k-m) !-1$ follows by Division algorithm. Then from part(ii) of Remark 2.2, it follows that, the leading coefficient of $L e(k ! l+r, k, m)-L e(k !(l-1)+r, k, m)$ is $(k-1) ! \frac{(k-m) !^{k-m-2}}{(k-m-1) !} \frac{k !^{k-m-1}}{(k-m) !^{k-m-1}}$. Consequently, the leading coefficient of Le $(k ! l+$ $r, k, m)$ is $(k-m-1)(k-1) ! \frac{(k-m) !^{k-m-2}}{(k-m-1) !} \frac{k !^{k-m-1}}{(k-m) !^{k-m-1}}$, which on simplification gives $\frac{(k-1) ! k !^{k-m-1}}{(k-m) !(k-m-2) !}$. Since $L e(k ! l+r, k, m)$ is a polynomial of degree $k-m-1$ for each $r=$ $0,1, \ldots, k !-1$, we get the estimate of $L e(n, k, m)$ as $\frac{(k-1) ! k !^{k-m-1}}{(k-m) !(k-m-2) ! k !^{k-m-1}} n^{k-m-1}$ $=\frac{(k-1) !}{(k-m) !(k-m-2) !} n^{k-m-1}$. The proof is now completed.

It is not hard to see that:

$$
q(n, k) \leq d(n, k) \leq p(n, k)
$$

Since $q(n, k) \sim \frac{n^{k-1}}{k !(k-1) !}$ and $p(n, k) \sim \frac{n^{k-1}}{k !(k-1) !}$, one can get the following estimate:

$$
d(n, k) \sim \frac{n^{k-1}}{k !(k-1) !} .
$$

Also, we see that:

$$
q(n, k) \leq L a(n, k, 1) \leq p(n, k) .
$$

Again, since $q(n, k) \sim \frac{n^{k-1}}{k !(k-1) !}$ and $p(n, k) \sim \frac{n^{k-1}}{k !(k-1) !}$, one can get the following estimate:

$$
\operatorname{La}(n, k, 1) \sim \frac{n^{k-1}}{k !(k-1) !} .
$$


Though estimates for $d(n, k)$ and $\operatorname{La}(n, k, 1)$ has been obtained immediately, it is the core objective of this article to show that $d(n, k)$ and $L a(n, k, 1)$ are quasi polynomials. We accomplish the same and thereby obtain the above mentioned estimates.

Theorem 2.4 We have

$$
d(n, k) \sim \frac{n^{k-1}}{k !(k-1) !} .
$$

Proof For instance, we call the partitions that $d(n, k)$ enumerates as deviated partitions. First, we prove the relation:

$$
d(n, k)=d(n-k, k)+p(n-1, k-1)
$$

when $n \geq k(k-1)+1$.

Let $\pi=\left(a_{1}, a_{2}, \ldots, a_{k}\right)$ be a deviated partition of $n$ with exactly $k$ parts. Now, we enumerate such partitions.

Case (i) Assume $a_{k}>1$. We notice that, the mapping

$$
\left(a_{1}, \ldots, a_{k}\right) \rightarrow\left(a_{1}-1, \ldots, a_{k}-1\right)
$$

establishes one to one correspondence between the following sets:

- The set of all deviated partitions of $n$ with exactly $k$ parts and least part being greater than one.

- The set of all deviated partitions of $n-k$ with exactly $k$ parts.

Case (ii) Assume $a_{k}=1$. Let $\pi=\left(a_{1}, \ldots, a_{k}\right)$ be a partition of $n$ with $a_{k}=1$. We note that: if $n \geq k(k-1)+1$ then $a_{1} \geq k$. Consequently, $\pi$ is a deviated partition of $n$ when $n \geq k(k-1)+1$. It is not hard to see that enumeration of such partitions is $p(n-1, k-1)$. Whence the relation (2.16).

From the relation (2.16) one can have the following relation:

$$
\begin{aligned}
d(k ! l+r, k)-d(k !(l-1)+r, k) & =\sum_{i=0}^{(k-1) !-1} p(k ! l-i k-1+r, k-1) \quad \text { when } l \geq 2 \\
& =\sum_{i=0}^{(k-1) !-1} p\left((k-1) !\left(k l+q_{i}\right)+r_{i}, k-1\right),
\end{aligned}
$$

where $\left(q_{i}, r_{i}\right)$ satisfying the inequality $0 \leq r_{i} \leq(k-1)$ ! -1 were uniquely determined from the relation $(k-1) ! q_{i}+r_{i}=r-i k-1$. From the above equality one can calculate the leading coefficient of $d(k ! l+r, k)$ to be $\frac{k !^{k-2}}{(k-1) !}$ for every $r=0,1, \ldots, k !-1$. Since $d(k ! l+r, k)$ is a polynomial of degree $k-1$ for every $r=0,1, \ldots, k !-1$, we get the estimate of $d(n, k)$ as $\frac{n^{k-1} k !^{k-2}}{k !^{k-1}(k-1) !}=\frac{n^{k-1}}{k !(k-1) !}$. This is what we wish to prove.

Theorem 2.5 We have

$$
\operatorname{La}(n, k, 1) \sim \frac{n^{k-1}}{k !(k-1) !}
$$


Proof We define $L a(n, k, M)$ to be the number of partitions of $n$ with exactly $k$ parts and $M$ largest parts. We show that

$$
L a(n, k, M)=L a(n-k, k, M)+L a(n-1, k-1, M) .
$$

Let $\pi=\left(a_{1}, \ldots, a_{k}\right)$ be a partition of $n$ with exactly $k$ parts and $M$ largest parts. Now, we count such partitions.

Case (i) Assume $a_{k}>1$. In this case, the mapping

$$
\left(a_{1}, \ldots, a_{k}\right) \rightarrow\left(a_{1}-1, \ldots, a_{k}-1\right)
$$

establishes one to one correspondence between the following sets:

- The set of all partitions of $n$ with $k$ parts, $M$ largest parts and least part being greater than 1.

- The set of all partitions of $n-k$ with $k$ parts and $M$ largest parts.

We see that, the cardinality of the latter set is $L a(n-k, k, M)$.

Case (ii) Assume $a_{k}=1$. In this case, the mapping

$$
\left(a_{1}, \ldots, a_{k}\right) \rightarrow\left(a_{1}, \ldots, a_{k-1}\right)
$$

establishes one to one correspondence between the following sets:

- The set of all partitions of $n$ with $k$ parts, $M$ largest parts and least part being one.

- The set of all partitions of $n-1$ with $k-1$ parts and $M$ largest parts.

Since cardinality of the latter set is $\operatorname{La}(n-1, k-1, M)$, above recurrence relation follows.

In this proof, we are concerned with the case $M=1$. We see that $\operatorname{La}(n, 1,1)=1$, $L a(2 l, 2,1)=q(2 l, 2)=l-1$ and $L a(2 l+1,2,1)=q(2 l+1,2)=l$. Thus, one can get inductively that: $\operatorname{La}(n, k, 1)$ is a quasi polynomial of degree $k-1$ with quasi period $k$ !. We show that the leading coefficient of the polynomial $L a(k ! l+r, k, 1)$ is $\frac{k !^{k-2}}{(k-2) !}$ for every $r=0,1, \ldots, k !-1$. By previous observation, the aforesaid assertion is true for $k=1,2$.

Assume that, the assertion is true up to some $k-1 \geq 1$. Let $a_{k-1}$ be the leading coefficient of $L a(k ! l+r, k, 1)$ for every $0 \leq r \leq k !-1$. We notice that, the leading coefficient of $L a(k ! l+r, k, 1)-L a(k !(l-1)+r, k, 1)$ is $(k-1) a_{k-1}$. By above recurrence relation, we have

$$
\begin{aligned}
& \operatorname{La}(k ! l+r, k, 1)-\operatorname{La}(k !(l-1)+r, k, 1) \\
& =\sum_{i=0}^{(k-1) !-1} \operatorname{La}\left((k-1) !\left(k l+q_{i}\right)+r_{i}, k-1,1\right),
\end{aligned}
$$

where $\left(q_{i}, r_{i}\right)$ were determined uniquely from the relation $(k-1) ! q_{i}+r_{i}=r-1-i$ with $0 \leq r_{i} \leq(k-1) !-1$. By the induction assumption, the leading coefficient of 
each of the polynomial in the right side of the above equality is $\frac{(k-1) !^{k-3} k^{k-2}}{(k-2) !}$. Thus the leading coefficient of $L a(k ! l+r, k, 1)-L a(k !(l-1)+r, k, 1)$ is $\frac{k !^{k-2}}{(k-2) !}$. This gives $a_{k-1}=\frac{k !^{k-2}}{(k-1) !}$.

Consequently, the estimate of $\operatorname{La}(n, k, 1)$ is $\frac{n^{k-1} k !^{k-2}}{(k-1) ! k^{k-1}}=\frac{n^{k-1}}{k !(k-1) !}$, as desired.

Open Access This article is distributed under the terms of the Creative Commons Attribution License which permits any use, distribution, and reproduction in any medium, provided the original author(s) and the source are credited.

\section{References}

1. Bell, E.T.: Interpolated denumerants and Lambert series. Am. J. Math. 65, 382-386 (1943)

2. David Christopher, A., et al.: Relatively prime uniform partitions. Gen. Math. Notes 13(2), 1-12 (2012)

3. Nathanson, M.B.: Partition with parts in a finite set. Proc. Am. Math. Soc. 128, 1269-1273 (2000)

4. Netto, E.: Le hrbuch der Combinatorik. Teubner, Leipzig (1927)

5. Rødseth, O.J., Sellers, J.: Partition with parts in a finite set. Int. J. Numb. Theory 02, 455 (2006)

6. Polya, G., Szegö, G.: Aufgaben and Lehrsätze aus der analysis. Springer, Berlin (1925) Problems and Theorems in Analysis, Springer-Verlag, New York, English translation 1972

7. Sertöz, S., Özlük, A.: On the number of representations of an integer by a linear form. İstanbul Üniv. Fen Fak. Mat. Derg. 50(1991), 67-77 (1993)

8. Wright, E.M.: A simple proof of a known result in partitions. Am. Math. Monthly 68, 144-145 (1961) 